\title{
ORIGINAL
}

\section{Las vacunas conjugadas y la enfermedad neumocócica en Ecuador}

\author{
E. Ortiz-Prado ${ }^{a, b, *}$, A.L. Iturralde ${ }^{d}$, P. Hernández ${ }^{b}$ y C. Galarza ${ }^{b, c}$ \\ a Facultad de Medicina, Universidad de las Américas, Quito, Ecuador \\ b Empresa Pública de Fármacos, ENFARMA EP, Quito, Ecuador \\ c Universidad de Cuenca, Cuenca, Ecuador \\ d Universidad Yachay, Urcuqui, Ecuador
}

\section{INFORMACIÓN DEL ARTÍCULO}

Historia del artículo:

Recibido el 16 de septiembre de 2014

Aceptado el 24 de octubre de 2014

On-line el 29 de octubre de 2014

\section{Palabras clave:}

Neumococo

Vacuna conjugada

Mortalidad

Neumonía

Ecuador

\begin{abstract}
R E S U M E N
Introducción: El Streptococcus pneumoniae es responsable de varios procesos infecciosos invasivos y no invasivos, especialmente en menores de 5 años y adultos mayores de 65 años. Las principales patologías asociadas a esta bacteria son: neumonía, otitis, bacteriemia, sepsis y meningitis. Según datos de la Organización Mundial de la Salud, un millón de niños mueren cada año a causa de estas enfermedades. En Ecuador, durante el 2011, la neumonía se convirtió en la primera causa de morbilidad en la población general y la segunda causa de mortalidad en la población infantil, constituyéndose en un problema de salud pública.

Objetivo: Identificar la vacuna antineumocócica más costo-efectiva para el Ecuador de acuerdo a su epidemiología.

Metodología: Se analizaron datos epidemiológicos de las bases de datos locales, regionales e internacionales, así como toda la evidencia disponible en relación con la enfermedad neumocócica.

Resultados: La vacuna tridecavalente (PCV13) tiene datos locales, regionales e internacionales que respaldan su uso e implementación en los cuadros de vacunación infantiles.

Conclusión: La estrategia actual de vacunación en el país no cubre todos los serotipos circulantes según los datos de SIREVA, de allí la importancia de ampliar la cobertura para estos serotipos vacunales otorgados por la vacuna tridecavalente.
\end{abstract}

(c) 2014 Publicado por Elsevier España, S.L.U.
Keywords:

Pneumococcus

Conjugate vaccine

Mortality

\section{Conjugate vaccines and pneumococcal disease in Ecuador}

\section{A B S T R A C T}

Introduction: Streptococcus pneumoniae is responsible for invasive and noninvasive infections in children under 5 and adults over 65 years, the pathologies associated with this pathogen are pneumonia, ear infections, bacteremia, sepsis and meningitis. According to the World Health Organization, one million children die every year due to this pathogen. In Ecuador

\footnotetext{
* Autor para correspondencia. Calle de los Colimes y Avenida De los Granados, Quito, Ecuador 170137

Correo electrónico: e.ortizprado@gmail.com (E. Ortiz-Prado).

http://dx.doi.org/10.1016/j.vacun.2014.09.002

1576-9887/@ 2014 Publicado por Elsevier España, S.L.U.
} 
Pneumonia

Ecuador in 2011, pneumonia was reported to be the first cause of morbidity in the general population and the second cause of death in children, becoming a public health problem.

Objective: To identify the most cost-effective anti pneumococcal vaccine in Ecuador according to our population needs.

Methodology: Analyzing data from local, regional and international databases as well as the latest evidence related with pneumococcal disease.

Results: The tridecavalente vaccine (PCV13) has local, regional and international data supporting its use and implementation in the child vaccination schedule.

Conclusion: Data from SIREVA suggest that current vaccination strategy in the country does not cover all circulating serotypes; hence, the importance of expanding serotypes coverage with the tridecavalente vaccine.

๑) 2014 Published by Elsevier España, S.L.U.

\section{Introducción}

Las infecciones por Streptococcus pneumoniae (S. pneumoniae) o neumococo continúan siendo una de las causas más importantes de morbimortalidad a nivel mundial ${ }^{1-3}$, así como las principales responsables de enfermedades invasivas en los últimos 20 años en el mundo sobre todo en niños menores de 5 años y adultos mayores, hecho agravado por la aparición de nuevos patrones de resistencia a los antibióticos de primera elección ${ }^{4,5}$.

Las formas de infección se clasifican en enfermedades invasivas (bacteriemia oculta, meningitis, y neumonía bacteriana) y no invasivas (otitis media aguda, neumonías, sinusitis y sobreinfecciones respiratorias) ${ }^{6}$..

En los países en vía de desarrollo, la enfermedad neumocócica invasiva (ENI) es uno de los determinantes de la morbimortalidad infantil, hecho que repercute con impacto en las economías de los países en vía de desarrollo por los costos que se generan al Sistema Nacional de Salud ${ }^{7}$.

En Ecuador, solo en el 2011 más de 48.000 pacientes fueron hospitalizados por estas causas (fig. 1). Si consideramos que un día de hospitalización en el sistema público de salud equivale a 700 USD diarios, el gasto por estos pacientes asciende a más de 33 millones de dólares en gastos hospitalarios ${ }^{8}$.

\section{Material y métodos}

Se realizó una revisión y análisis estructurado de la literatura publicada hasta la fecha en bases de datos electrónicas (Medline, Embase, Cochrane Library Database e Inbiomed) así como las fuentes de datos oficiales disponibles en el país (Instituto Nacional de Estadísticas y Censos, Ministerio de Salud Pública); cuando no fue posible obtener datos específicos propios se consideraron fuentes referidas al contexto latinoamericano y a la ausencia de estas fuentes internacionales.

\section{El neumococo y sus serotipos}

Se han identificado al menos 90 serotipos de S. pneumoniae; actualmente no se puede realizar una vacuna con todos las cepas, aunque se han hecho varias vacunas que cubren el 8590\% de las causas de enfermedad neumocócica ${ }^{9}$.
Los serotipos 1, 5, 6A, 6B, 14, 19F y 23F se constituyen en la causa frecuente de enfermedad neumococcica en menores de 5 años en todo el mundo ${ }^{10}$. En los últimos años se han producido cambios indiscutibles en la epidemiología de la ENI, debido a que la presión antibiótica favorece la selección de cepas resistentes y por ende el tratamiento y la prevención se hacen cada vez más complicados ${ }^{11}$.

De los posibles serotipos con mayor índice de resistencia tenemos a los 6B, 9V, 14, 19A, 19F y 23. Por otro lado, existe un reemplazo de los serotipos colonizadores, dando lugar a un incremento de enfermedad neumocócica por serotipos no prevenibles $^{11}$.

En Ecuador, en el 2011, la neumonía es la primera causa de morbilidad general, generando 34.778 hospitalizaciones ${ }^{12,13}$; de estos casos 18.153 corresponden a la población infantil menor de 5 años, siendo 7.147 casos niños menores de un año y 11.006 niños entre 1-4 años, constituyéndose en la segunda causa de mortalidad infantil, luego de causas relacionadas con el parto y el nacimiento ${ }^{13}$.

En este contexto, Ecuador ha implementado la vacunación sistemática y progresiva, comenzando con vacuna conjugada neumocócica heptavalente (PCV7) desde el año 2008 para los grupos de riesgo, y para el 2010 su inclusión en el calendario nacional de inmunización. En marzo del 2011 se implementó la vacuna conjugada neumocócica decavalente (PCV10) la misma que se mantiene hasta la actualidad ${ }^{14}$.

Ecuador es parte de la red del Sistema de Redes de Vigilancia de los Agentes Bacterianos Responsables de Neumonía

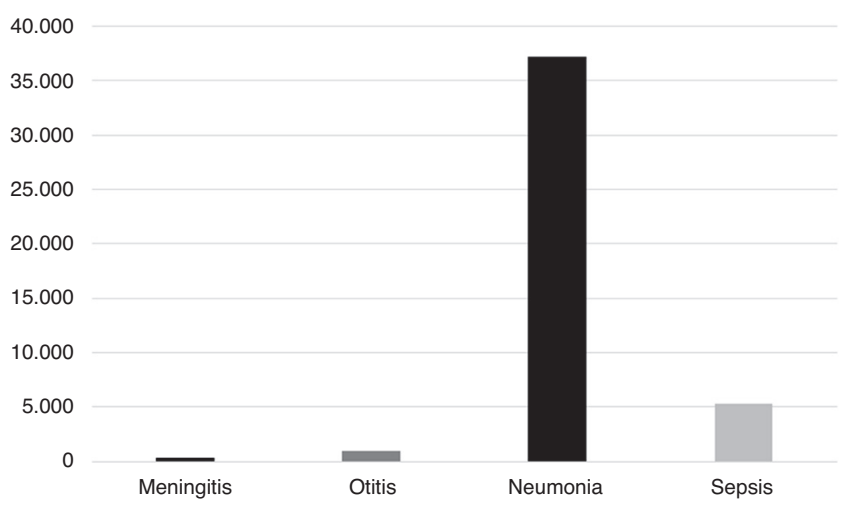

Figura 1 - Morbilidad Hospitalaria por Streptococcus pneumoniae reportada en Ecuador en el 2011. 


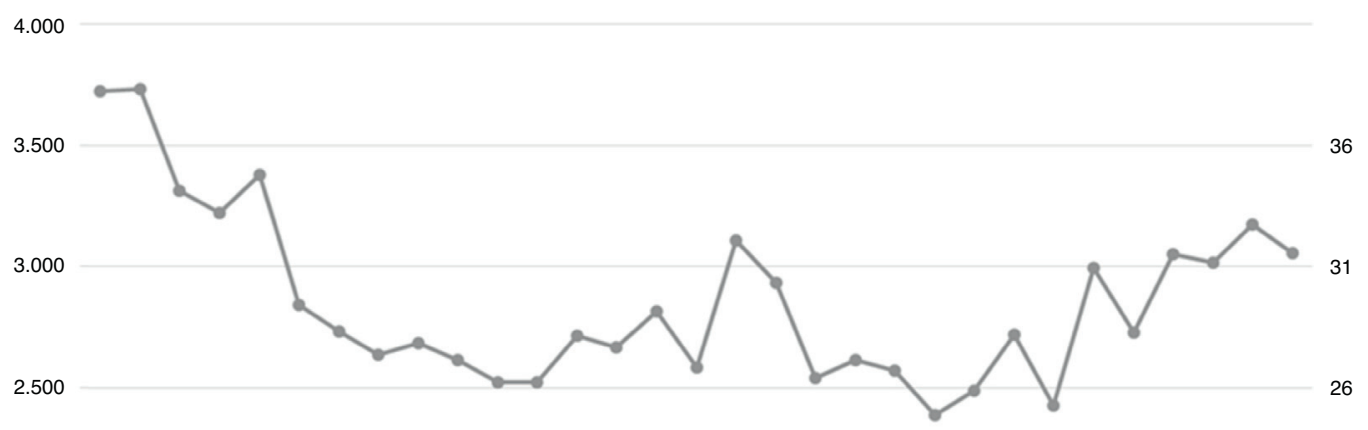

2.000

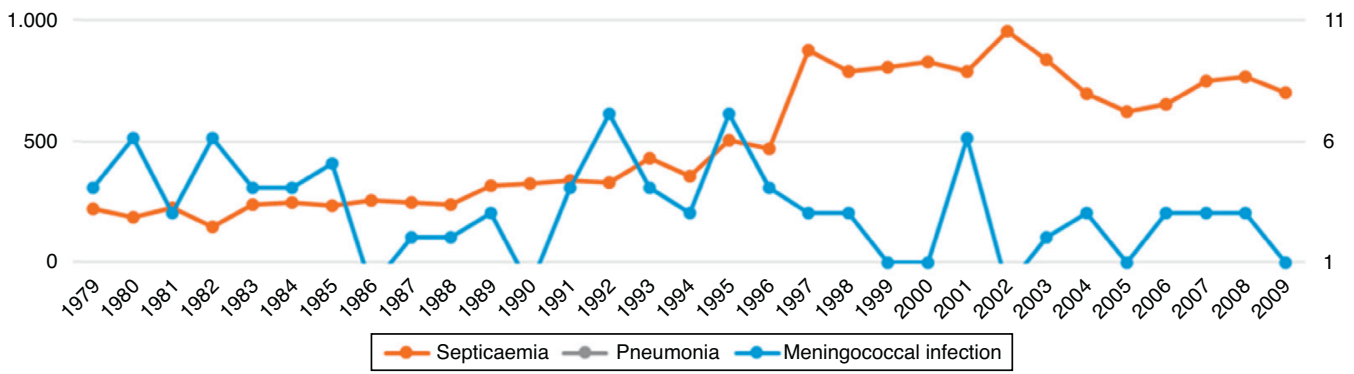

Figura 2 - Enfermedad Neumocócica en Ecuador en el año 2011.

y Meningitis, SIREVA desde 1994, con 18 años de información disponible con relación al comportamiento de los serotipos provenientes de aislamientos invasivos de $S$. pneumoniae provenientes de diferentes regiones del país (fig. 2).

En el Informe Regional de SIREVA II-2011 se observa una elevación importante de los serotipos no cubiertos por la vacuna PCV7 que actualmente se utiliza en el país frente a la información reportada por el SIREVA II-2010 para estos mismos serotipos durante el mismo período de tiempo ${ }^{15}$ (tabla 1).

La elevación de estos serotipos adquiere importancia clínica significativa debido a sus condiciones propias; por ejemplo el serotipo 3, es relativamente frecuente en casos de ENI, otitis media aguda ${ }^{16}$ y está asociado con neumonía complicada; el serotipo 6A es una importante causa de ENI asociada a resistencia bacteriana ${ }^{17}$, y, el serotipo 19A es el serotipo de mayor incremento en la región. En la tabla 1 se puede evidenciar el comportamiento de estos serotipos antes de la vacunación y después de la misma en nuestro país.

La dinámica de los serotipos no cubiertos por la vacuna que actualmente utiliza el Programa Nacional de Inmunización hace que en el país circulen serotipos no cubiertos por esta intervención, similar a lo que se reporta en otros países antes de la inclusión de una PCV10 de mayor cobertura ${ }^{18}$; este es un elemento de gran importancia a considerar así como la carga de enfermedad asociada a estos serotipos, la cual se podría prevenir con una vacuna de mayor cobertura, lo que representaría una reducción de la mortalidad en menores de 5 años y costos para el Sistema Nacional de Salud de Ecuador.

\section{Tipos de vacunas antineumocócicas}

En la actualidad se comercializan 2 tipos diferentes de vacunas antineumocócicas que son:

1) La vacuna polisacarídica 23-valente (PPV23) que está disponible desde principios de los años ochenta y se usa principalmente en adultos mayores de 65 años de edad.

2) Las vacunas conjugadas, que son la vacuna decavalente (PCV10), la tridecavalente (PCV13) y la vacuna conjugada heptavalente (PCV7) que se está retirando gradualmente del mercado ${ }^{19}$.

\section{Aspectos farmacoeconómicos}

Para la elección de una u otra estrategia de salud pública es indispensable realizar un análisis minucioso y de costo/beneficio mediante un análisis farmacoeconómico de las vacunas disponibles (PCV10-PCV13) como instrumento de la toma de decisión más acertada a implementarse como parte del Programa Ampliado de Inmunizaciones Nacional.

En este tipo de análisis cobran vital importancia los resultados que se relacionan con años de vida ajustados a calidad, muertes evitadas, y la reducción de casos de enfermedad y los costos directos, indirectos e intangibles generados ${ }^{20,21}$.

Para la aplicación de la efectividad de las vacunas conjugadas en la cobertura local de serotipos a fin de estimar los casos prevenibles por PCV10-PCV13, se tomaron en cuenta los 
Tabla 1 - Datos históricos SIREVA II 2000-2011, correspondientes a Ecuador ${ }^{15}$

\begin{tabular}{|c|c|c|c|c|c|c|c|}
\hline \multicolumn{8}{|c|}{ Ecuador } \\
\hline Serotipo & $\begin{array}{l}2000-05 \\
n=55\end{array}$ & $\begin{array}{l}2006 \\
n=38\end{array}$ & $\begin{array}{l}2007 \\
n=41\end{array}$ & $\begin{array}{l}2008 \\
n=36\end{array}$ & $\begin{array}{l}2009 \\
n=42\end{array}$ & $\begin{array}{l}2010 \\
n=35\end{array}$ & $\begin{array}{l}2011 \\
n=36\end{array}$ \\
\hline 1 & 4 & 9 & 5 & 2 & 1 & 5 & 3 \\
\hline 3 & 0 & 1 & 0 & 0 & 4 & 0 & 1 \\
\hline 4 & 0 & 0 & 0 & 2 & 0 & 0 & 0 \\
\hline 5 & 3 & 3 & 5 & 2 & 1 & 0 & 0 \\
\hline $6 \mathrm{~A} / 6 \mathrm{C}$ & 3 & 1 & 2 & 1 & 2 & 0 & 3 \\
\hline $6 \mathrm{~B}$ & 7 & 3 & 8 & 0 & 3 & 5 & 3 \\
\hline $7 F$ & 0 & 1 & 0 & 0 & 1 & 0 & 1 \\
\hline $9 \mathrm{~V}$ & 2 & 2 & 0 & 1 & 4 & 4 & 1 \\
\hline 14 & 17 & 6 & 12 & 12 & 15 & 9 & 13 \\
\hline $18 \mathrm{C}$ & 1 & 0 & 1 & 0 & 0 & 0 & 1 \\
\hline $19 \mathrm{~A}$ & 0 & 1 & 1 & 0 & 2 & 5 & 7 \\
\hline $19 \mathrm{~F}$ & 3 & 2 & 1 & 0 & 3 & 4 & 3 \\
\hline $23 \mathrm{~F}$ & 3 & 1 & 0 & 0 & 4 & 1 & 0 \\
\hline Otros & 12 & 8 & 6 & 16 & 2 & 2 & 0 \\
\hline Total & 55 & 38 & 41 & 36 & 42 & 35 & 36 \\
\hline
\end{tabular}

efectos directos: basados en la efectividad reportada de las 2 vacunas y ajustada a la epidemiología $\operatorname{local}^{9}$; y los efectos indirectos o rebaño: basados en la data del sistema de vigilancia de salud fundamentada en $\mathrm{PCV}^{26}$. Estos datos, estimados para una cohorte de nacimientos de 267.000 nacidos vivos, de una población total de 14,8 millones, según el último censo reportado por el INEC en $2012^{13}$.

Los datos de incidencia, fatalidad y costo por manejo de casos, se hicieron en función de los informes SIREVA II 2010-2011 y de reportes epidemiológicos nacionales e internacionales ${ }^{1,15,22}$ (tabla 1).

La data epidemiológica fue estimada a partir de los recursos internacionales. Los rangos de incidencia y mortalidad de ENI en niños de 0-4 años fueron aplicados a partir de la estimación de O’Brien et al., estimados para Ecuador ${ }^{1}$ (fig. 3).

Todas las causas de neumonía fueron tomadas de los estimados derivados del Child Health Epidemiology Reference
Group, donde se asume que el $8.6 \%$ de los casos severos de neumonía requieren de hospitalización. El costo por episodio fue estimado según los costos médicos directos publicados en Nakamura et al. ${ }^{23}$. Los costos médicos se han ajustado al país basados en el gasto en salud per cápita reportados en el 2005 e incrementados al 2011 en un rango proporcional de crecimiento para el país desde el 2005 al 2011.

Los costos estimados en el tratamiento para meningitis fueron de 6.750 USD, para bacteriemia \$1.326USD, para neumonía intra y extra hospitalaria 2.182 y 56 USD respectivamente ${ }^{24}$.

Los costos de la PCV10-PCV13 son los ofrecidos por el Fondo Rotatorio de OPS más 1 USD por la administración de la vacuna PCV10 = 14.12 USD y PCV13 = 15.84 USD. El modelo de vacunación se asume en 3 dosis (2 dosis + una de refuerzo) como lo mantiene nuestro programa ampliado de inmunización nacional $^{25}$ (tabla 2).

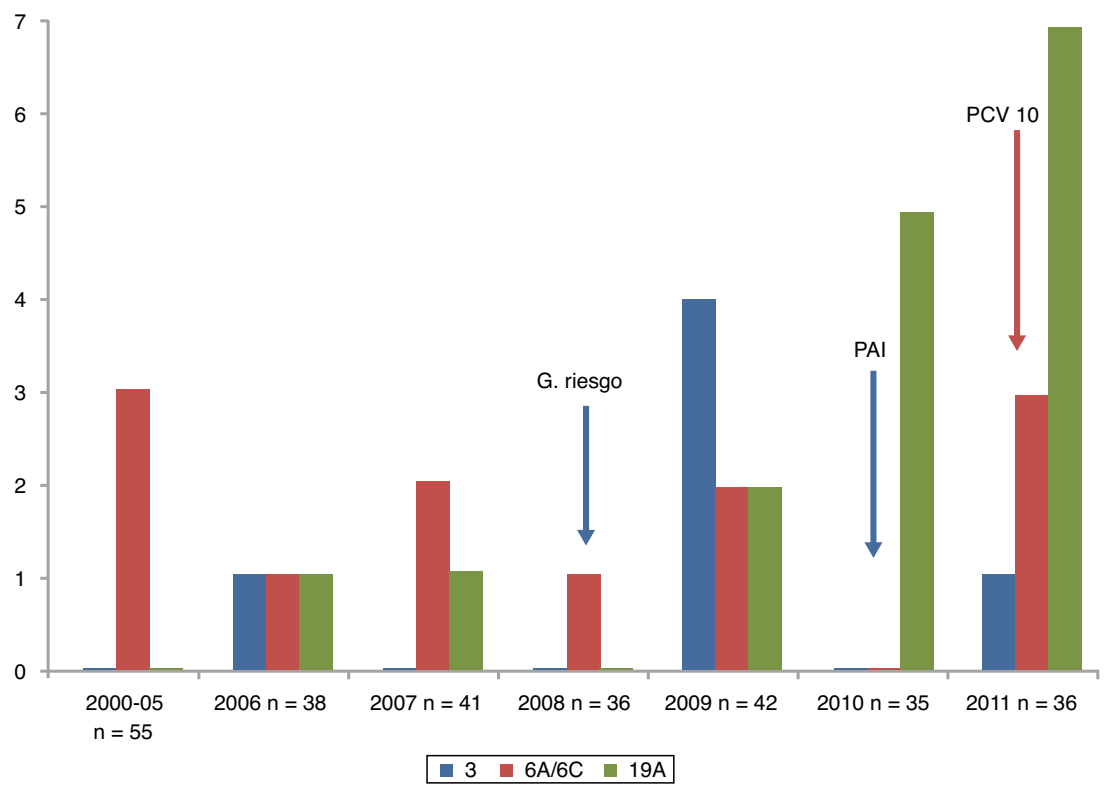

Figura 3 - Comportamiento de los serotipos 3, 6 a / 6 c y 19a desde 2000 al 2011. 
Tabla 2 - C omparación de costos entre la intervención con PCV10 y PCV13 en Ecuador

\begin{tabular}{|c|c|c|c|}
\hline Intervención & PCV10 & PCV13 & PCV13-PCV10 \\
\hline \multicolumn{4}{|l|}{ Costos ahorrados por enfermedad } \\
\hline ENI & 4.557.114 USD & 5.445.911 USD & 888.797 USD \\
\hline Neumonía hospitalaria & 11.204.267 USD & 13.104.842 USD & 1.900.575 USD \\
\hline Neumonía ambulatoria & 1.175.613 USD & 1.321.687 USD & 146.074 USD \\
\hline OMA & 3.865.860 USD & 4.321.493 USD & 455.633 USD \\
\hline Total & 20.802.854 USD & 24.193.933 USD & 3.391.079 USD \\
\hline \multicolumn{4}{|l|}{ Inversión PAI } \\
\hline Total & 10.900.865 USD & 12.140.910 USD & 1.240.045 USD \\
\hline \multicolumn{4}{|l|}{ Evaluación de la intervención } \\
\hline Costo neto ahorrado al SNS & 9.901.989 USD & 12.053.023 USD & 2.151.034 USD \\
\hline Muertes evitadas & 396 & 468 & 72 \\
\hline Años de vida ganados & 8.512 & 10.025 & 1.513 \\
\hline Años de vida ajustados a calidad ganados & 8.020 & 9.442 & 1.421 \\
\hline
\end{tabular}

Costos prevenibles: PCV13 mediante la cobertura de los 3 serotipos adicionales, se prevendrían 323 casos potenciales de ENI; 3.480 casos de neumonía y 8.136 casos de otitis media aguda por año, con relación a la PCV10 donde no se incluyen estos serotipos.

Costos evitados: La cobertura adicional de los 3 serotipos 3, 6A y 19A por la PCV13 evitaría el gasto de 3,4 millones de dólares en costos médicos directos comparados con la PCV10 en la reducción de la enfermedad neumocócica.

La efectividad de las vacunas se ha obtenido de estudios clínicos ajustados a la cobertura local de PCV10-PCV13. La cobertura de serotipos para cada vacuna se aplicó del promedio reportado por Ecuador al SIREVA mediante el Sistema de Vigilancia de los hospitales centinela.

\section{Discusión}

Es incuestionable el impacto global en positivo que se ha generado en relación con la reducción de la carga de enfermedad por los serotipos vacunales en países donde se ha incluido esta vacuna de forma sistemática, hecho que se ha acompañado de un incremento significativo de la enfermedad neumocócica por serotipos no incluidos en la misma; las razones de este comportamiento epidemiológico se han atribuido al cambio en la colonización nasofaríngea por efecto de la vacunación y por el uso irracional de antibióticos lo que sin duda influye en el incremento de serotipos multirresistentes ${ }^{26}$.

No hay estudios publicados sobre comparaciones directas de la PCV10 y la PCV13, y hasta ahora solo hay un estudio de efectividad con la PCV13, en Inglaterra y Gales, donde la PCV7 se sustituyó por la PCV13 en abril de 2010. Las ENI por serotipos presentes en la PCV13 se redujeron a la mitad durante el período de estudio en los menores de 2 años ${ }^{27}$.

La eficacia de la vacunación para prevenir la neumonía se estimó a partir de la literatura. Se asumió que la PCV10 y la PCV13 evitarían un 4,3\% de los casos ambulatorios. Para las neumonías que requieren hospitalización se estimó que la PCV10 evitaría un 40\% de los casos, frente a un $46 \%$ de la PCV13. El porcentaje de casos de otitis media aguda neumocócica cubiertos por los serotipos incluidos en la PCV10 y la PCV13 se estimó en un $71,6 \%$ y en un $82,2 \%$, respectivamente $23,26,28$.
Todo esto, de acuerdo a los resultados de un estudio realizado por el Ministerio de Salud del Perú el cual concluye que las intervenciones con PCV10 y PCV13 demuestran ser más costo-efectivas en la reducción de hospitalizaciones por neumonía que la estrategia con PCV. A su vez concluye que la PCV13 es más costo-efectiva que la PCV10 ${ }^{29}$.

En general, los resultados de estas investigaciones sugieren que un programa de inmunización basado en la PCV13 representa una opción más efectiva y menos costosa que programas basados en la PCV7 y la PCV10 ${ }^{30}$.

Bajo este argumento, se debe considerar además que la PCV7 y la PCV13 contienen el mismo carrier proteico por lo que son totalmente intercambiables, de manera que cualquier niño en el que se haya iniciado la vacunación con PVC7 podrá continuar con PCV13 hasta completar el esquema de inmunización ${ }^{31}$.

Los niños que han iniciado la vacunación con PCV10 deben completar toda la serie de inmunización (primovacunación y refuerzo) con la PCV10, pues hasta el momento no existen datos que documenten la intercambiabilidad, ni la transición a la PCV13 32

Los niños de hasta 59 meses de edad que previamente hayan recibido toda la serie de inmunización completa con cualquiera de las vacunas antineumocócicas conjugadas PCV7 o PCV10 pueden beneficiarse de una dosis adicional de PCV13, administrada con al menos 2 meses de intervalo tras la última dosis de vacuna antineumocócica; esta estrategia de inmunización permite ampliar su protección a los serotipos que incorpora esta nueva vacuna ${ }^{26}$.

En niños y adolescentes de 6 a 17 años, la incidencia de la enfermedad neumocócica es baja; sin embargo, existe un mayor riesgo de morbimortalidad en los pacientes con comorbilidades, personas mayores que viven en instituciones cerradas y en otros grupos de riesgo en los cuales se recomienda la aplicación de una vacuna conjugada y a las 8 semanas una dosis de vacuna antineumocócica no conjugada $(\mathrm{PPV} 23)^{25}$.

La PPV23 incluye los serotipos 1, 2, 3, 4, 5, 6B, 7F, 8, 9N, 9V, 10A, 11A, 12F, 14, 15B, 17F, 18C, 19A, 19F, 20, 22F, 23F y 33F; esta vacuna estimula el sistema inmune $\mathrm{T}$ independiente por lo que no son inmunógenas en niños pequeños y no generan respuestas mucosas, o son muy débiles, ni memoria inmunológica. La respuesta humoral es fundamentalmente de IgG, IgA 
e IgM frente a la enfermedad invasiva y a la neumonía de todas las causas en adultos jóvenes sanos y en menor medida en ancianos frente a enfermedad invasiva. Los ensayos clínicos no han podido demostrar eficacia frente a ENI o neumonía de todas las causas en individuos inmunodeprimidos, y muchos estudios observacionales sugieren una efectividad entre el 50$80 \%$ frente a ENI en adultos sanos ${ }^{33}$.

Para la vacunación en niños mayores de 24 meses que presenten riesgo o comorbilidades y que hayan completado el esquema con PCV13, se recomienda la administración de la vacuna PPV23 ${ }^{9}$.

\section{Conclusiones}

Revisada la literatura disponible y las recomendaciones emitidas por OMS/OPS y la Sociedad Latinoamericana de Infectología Pediátrica (SLIPE) y analizados los datos estadísticos de los informes SIREVA II, se recomienda que en Ecuador se tomen en consideración los serotipos aislados para establecer los esquemas de vacunación que más favorezcan a la salud de las y los ecuatorianos.

De la literatura revisada se puede inferir que el fondo gubernamental invertido en programas de inmunización es altamente costo-efectivo o costo-ahorrativo, particularmente cuando la reducción de la enfermedad en sujetos no vacunados (efecto rebaño) es considerada, razón por la cual el efecto rebaño incrementa significativamente el valor de la vacunación a través del ahorro de costos y vidas en no vacunados; bajo esta premisa la PCV13 es la que ha demostrado tener este efecto, por lo cual es importante considerar el análisis a fondo de las variables epidemiológicas y farmacoeconómicas a fin de tomar una adecuada decisión basada en la realidad actual del país. En este contexto es primordial e indispensable que en Ecuador se cuente con una vacuna que cubra todos los serotipos aislados en el medio; el costo adicional para vacunar con la PCV13 se ve compensado por el ahorro asociado a la reducción de enfermedades neumocócicas, en comparación con la PCV10.

\section{Autoría}

Este manuscrito ha sido elaborado por el Dr. Esteban OrtizPrado y la Dra. Ana Lucía Iturralde con la colaboración de la Dra. Paulina Hernández en lo referente a la obtención y análisis de datos; y la dirección ha estado a cargo del investigador Claudio Galarza.

\section{Financiación}

Este manuscrito no recibió financiamiento alguno.

\section{Conflicto de intereses}

Nosotros no tenemos ningún tipo de conflicto de interés, la información es este manuscrito es netamente objetiva y científicamente validada.
BIBLIOGRAF Í A

1. O’Brien KL, Wolfson LJ, Watt JP, Henkle E, Deloria-Knoll M, McCall N, et al. Burden of disease caused by Streptococcus pneumoniae in children younger than 5 years: Global estimates. Lancet. 2009;374:893-902.

2. Robinson KA, Baughman W, Rothrock G, Barrett NL, Pass M, Lexau C, et al. Epidemiology of invasive Streptococcus pneumoniae infections in the United States, 1995-1998: Opportunities for prevention in the conjugate vaccine era. JAMA. 2001;285:1729-35.

3. Gordon MA, Walsh AL, Chaponda M, Soko D, Mbvwinji M, Molyneux ME, et al. Bacteraemia and mortality among adult medical admissions in Malawi-Predominance of non-typhi Salmonellae and Streptococcus pneumoniae. J Infect. 2001;42:44-9.

4. Cueto Montoya GA, Pérez Cueto MC. Streptococcus pneumoniae aislados de infecciones invasivas: serotipos y resistencia antimicrobiana. Rev Cuba Med Gen Integral. 2007;23:0.

5. Toraño Peraza GT, Llanes Caballero R, Pías Solis LM, Abreu Capote M, Valcárcel Sánchez M. Serotipos de Streptococcus pneumoniae en Cuba y progresión de la resistencia a la penicilina. Rev Cubana Med Trop. 2010;62:157-60.

6. Bogaert D, de Groot R, Hermans PWM. Streptococcus pneumoniae colonisation: The key to pneumococcal disease. Lancet Infect Dis. 2004;4:144-54.

7. Constenla DO. Economic impact of pneumococcal conjugate vaccination in Brazil, Chile, and Uruguay. Rev Panam Salud Pública. 2008;24:101-12.

8. Subsecretaría Nacional de Gobernanza de la Salud Pública [Internet]. Ministerio de Salud Pública [citado 31 Mar 2014]. Recuperado a partir de: http://www.salud.gob.ec/ subsecretaria-nacional-de-gobernanza-de-la-salud-publica/

9. Musher DM. Pneumococcal vaccination in adults [Internet]. Up To date. 2014. Recuperado a partir de: http://www. uptodate.com.ezproxy.lib.ucalgary.ca/contents/ pneumococcal-vaccination-in-adults? source=search result\&search=pneumococcal+vaccine\&selectedTitle $=1 \sim 150$

10. Hicks LA, Harrison LH, Flannery B, Hadler JL, Schaffner W, Craig AS, et al. Incidence of pneumococcal disease due to non-pneumococcal conjugate vaccine (PCV7) serotypes in the United States during the era of widespread PCV7 vaccination, 1998-2004. J Infect Dis. 2007;196:1346-54.

11. Kyaw MH, Lynfield R, Schaffner W, Craig AS, Hadler J, Reingold $A$, et al. Effect of introduction of the pneumococcal conjugate vaccine on drug-resistant Streptococcus pneumoniae. N Engl J Med. 2006;354:1455-63.

12. INEC. Ecuador en cifras. Anuario de estadísticas hospitalarias: camas y egresos 2011 [Internet]. 2011 [consultado 2014]. Recuperado a partir de: www.inec.gob.ec

13. INEC. Ecuador en cifras. Anuario de nacimientos y defunciones 2011. Principales causas de morbilidad general [Internet]. 2012 [consultado 2014]. Recuperado a partir de: www.inec.gob.ec

14. MSP. Ministerio de Salud Publica del Ecuador. Datos del programa ampliado de inmunizaciones [Internet]. 2012 [consultado 2014]. Recuperado a partir de: www.salud.gob.ec

15. PAHO. Vigilancia sSanitaria, prevención y control de enfermedades (HSD). Regulaciones sanitarias internacionales, alerta y respuesta y enfermedades epidémicas (HSD/IR). Informe Regional de SIREVA II, 2011: Datos por país y por grupos de edad sobre las características de los aislamientos de Streptococcus pneumoniae, Haemophilus influenzae y Neisseria meningitidis en procesos invasores. 2012.

16. Shinefield H, Black S, Ray P, Fireman B, Schwalbe J, Lewis E. Efficacy, immunogenicity and safety of heptavalent 
pneumococcal conjugate vaccine in low birth weight and preterm infants. Pediatr Infect Dis J. 2002;21:182-6.

17. Hansen J, Black S, Shinefield H, Cherian T, Benson J, Fireman $B$, et al. Effectiveness of heptavalent pneumococcal conjugate vaccine in children younger than 5 years of age for prevention of pneumonia: updated analysis using World Health Organization standardized interpretation of chest radiographs. Pediatr Infect Dis J. 2006;25: 779-81.

18. Bingen E, Levy C, Varon E, de la Rocque F, Boucherat M, d'Athis P, et al. Pneumococcal meningitis in the era of pneumococcal conjugate vaccine implementation. Eur J Clin Microbiol Infect Dis. 2008;27:191-9.

19. Control C for D (CDC P. Use of 13-valent pneumococcal conjugate vaccine and 23-valent pneumococcal polysaccharide vaccine for adults with immunocompromising conditions: Recommendations of the Advisory Committee on Immunization Practices (ACIP). MMWR Morb Mortal Wkly Rep. 2012;61:816.

20. Baly Gil A, Toledo ME, Rodríguez Jústiz F. La economía de la salud, la eficiencia y el costo de oportunidad. Rev Cuba Med Gen Integral. 2001;17:395-8.

21. Consuelo DV. Coste-efectividad de la vacunación neumocócica. Gac Sanit. 1999;13:9054.

22. Liu L, Johnson HL, Cousens S, Perin J, Scott S, Lawn JE, et al. Global, regional, and national causes of child mortality: An updated systematic analysis for 2010 with time trends since 2000. Lancet. 2012;379:2151-61.

23. Nakamura MM, Tasslimi A, Lieu TA, Levine O, Knoll MD, Russell LB, et al. Cost effectiveness of child pneumococcal conjugate vaccination in middle-income countries. Int Health. 2011;3:270-81.

24. MSP. Tarifario prestaciones del Sistema Nacional de Salud. 2012.

25. Urueña A, Pippo T, Betelu MS, Virgilio F, Giglio N, Gentile A, et al. Cost-effectiveness analysis of the 10-and 13-valent pneumococcal conjugate vaccines in Argentina. Vaccine. 2011;29:4963-72.

26. Rubió PP, Morales PG, Sanmartí LS. Coste-efectividad de la vacunación neumocócica en Cataluña. Rev Esp Salud Pública [Internet]. 1995; 69 [citado $27 \mathrm{Feb}$ 2014]. Recuperado a partir de: http://www.msc.es/biblioPublic/publicaciones/ recursos_propios/resp/revista_cdrom/VOL69/69_5_409.pdf

27. Johnson HL, Deloria-Knoll M, Levine OS, Stoszek SK, Hance LF, Reithinger R, et al. Systematic evaluation of serotypes causing invasive pneumococcal disease among children under five: The pneumococcal global serotype project. PLoS Med. 2010;7:e1000348.

28. Larraz GG, Ortiz HT, Mourine NS, Giglio N, Cané A, García $\mathrm{MCP}$, et al. Costo-efectividad de la vacunación universal antineumocócica en Uruguay. Rev Panam Salud Publica. 2010;28:92-9.

29. Bolaños Díaz R, Mezones-Holguín E, Fiestas Solórzano V, Suárez Moreno V, Sanabria C. Evaluación económica de las vacunas hepta-, deca-y trece-valente para la prevención de neumonía asociada al Streptococcus pneumoniae en niños menores de 5 años en el Perú. Lima: UNAGESP; 2011.

30. Muciño-Ortega E, Mould-Quevedo JF, Farkouh R, Strutton D. Evaluación económica de un programa de inmunización infantil en México basado en la vacuna neumocócica conjugada 13-valente. Value Health. 2011;14:S65-70.

31. AEP. Comité Asesor de Vacunas de la Asociación Española Pediatría. Informe del comité asesor de vacunas de la comunidad de Madrid sobre la transición de la vacunación antineumocócica infantil. 2010; p. 1-10.

32. Kaye P, Malkani R, Martin S, Slack M, Trotter C, Jit M, et al. Invasive pneumococcal disease (IPD) in England \& Wales after 7-valent conjugate vaccine (PCV7); potential impact of 10 and 13-valent vaccines. Póster Presentado en: the 27th Annual Meeting of the European Society for Paediatric Infectious Disease, Brussels [Internet]. 2009; p.13 [citado 27 Feb 2014]. Recuperado a partir de: http://meetings.espid.org/ espid09/abstracts/pdf/832.pdf

33. Nuorti JP, Whitney CG. Prevention of pneumococcal disease among infants and children: Use of 13-valent pneumococcal conjugate vaccine and 23-valent pneumococcal polysaccharide vaccine: Recommendations of the Advisory Committee on Immunization Practices (ACIP) [Internet]. Department of Health and Human Services, Centers for Disease Control and Prevention; 2010 [citado 27 Feb 2014]. Recuperado a partir de: http://www.cdc.gov/mmwr/ preview/mmwrhtml/rr5911a1.htm?s_cid\%3Drr5911a1_x 